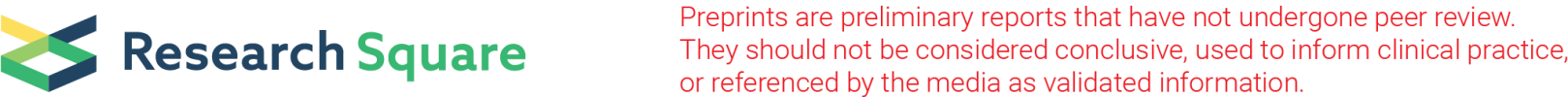

\section{Empiric and Conservative Strategy in The Interventional Management of Late Post- Pancreatoduodenectomy Hemorrhage With Negative Angiographic Result}

\section{Qiang Huang}

Beijing Chaoyang Hospital, Capital Medical University https://orcid.org/0000-0002-5949-1225

\section{Kun Gao}

Beijing Chaoyang Hospital

Jian-Feng Wang ( $\square$ jianfeng_wang@126.com )

Capital Medical University https://orcid.org/0000-0002-2409-0544

\section{Research article}

Keywords: post pancreaticoduodenectomy hemorrhage, empiric embolization, angiography, relaparotomy

Posted Date: December 30th, 2020

DOI: https://doi.org/10.21203/rs.3.rs-136104/v1

License: (c) (1) This work is licensed under a Creative Commons Attribution 4.0 International License.

Read Full License 


\section{Abstract}

Background: The aim of the study was to review the outcome of patients who underwent interventional procedures with negative angiographic results for post-PD hemorrhage. Empiric and conservative interventional strategies were compared.

Methods: The consecutive patients who underwent interventional procedures for post-PD hemorrhage in our center between Jan 2016 and Jun 2020 were evaluated. 21 cases were enrolled into this study with negative angiographic results. Two different strategies, empiric and conservative, were applied. Clinical data, including age, sex, pathological diagnosis, lab test results, clinical presentation and onset time of bleeding after surgery, technical and clinical outcome was obtained from the medical records and follow up data.

Results: All patients in our series presented with delayed post-PD hemorrhage. In the empiric group, embolization was performed at the hepatic artery in 11 cases and at the left gastric artery (LGA) in 1 . Two patients died of hemorrhage recurrence despite embolization during the follow up. Two patients required laparotomy and recovered in this group. Recurrence rate was $33.3 \%(4 / 12)$ in this group and mortality rate was $16.7 \%(2 / 12)$. In the conservative group, one patient required re-angiography with bleeding from the hepatic artery revealed 10 days after the first angiography. Hepatic artery embolization and subsequent relaparotomy was required. Another two patients required relaparotomy for hemorrhage recurrence. Recurrence rate was $33.3 \%$ (3/9) in this group and all these 3 cases required relaparotomy as definite treatment.

Conclusion: Prompt decision making is required when negative result demonstrated during the angiography for post PD hemorrhage, and the surgeons' judgement is mandatory. Both empiric and conservative treatment may be effective as indicated when negative angiography presented. Great caution is required following the interventional procedure, because recurrence rate after both treatment methods is significant despite negative angiography.

\section{Background}

Post-pancreatoduodenectomy hemorrhage is one of the fatal and life-threatening complications of pancreaticoduodenectomy (PD), or referred to as the Whipple operation, and its occurrence still results in high mortality $(1,2)$. The incidence of postoperative hemorrhage is about $4 \%-16 \%$, and the mortality is as high as $11 \%-54 \%(3)$.

Interventional procedure has been proved to be an effective preferred or first-line treatment for delayed post PD hemorrhage, reducing the need for immediate relaparotomy $(1,4-10)$. However, positive findings are not always revealed on angiography. There is no consensus on the optimal management when negative angiography presented. Empiric embolization despite negative angiography had been reported $(11-13)$, but it has not yet been widely advocated. Therefore, a detailed indication for empiric embolization for negative angiography has still to be established. To determine the current role of empiric 
embolization and conservative treatment following negative angiography, as well as the risks and outcomes, we retrospectively reviewed our experience in the treatment of 21 patients with negative angiography for post PD hemorrhage.

\section{Methods}

The consecutive patients who underwent interventional procedures for post-PD hemorrhage in our center between Jan 2016 and Jun 2020 were evaluated. 21 cases were enrolled into this study with negative angiographic results. Written informed content was waived because of the retrospective design of this study in our center. Post PD hemorrhage was detected by the presence of fresh blood in the surgical drains or nasogastric tube, hematemesis or melena. The hemorrhage was categorized according to the criteria formulated by the International Study Group of Pancreatic Surgery (ISGPS) (14).

Written informed consent was obtained before the interventional procedures in all the patients. All endovascular treatments were performed by one of the five senior interventional radiologists with at least 5 years of experience. Interventional procedures were performed using the Allura Xper FD20 system (Philips, Amsterdam, the Netherlands). Vascular access was achieved with a 5-F sheath introduced in the right femoral artery. Angiography was performed with a 5-F catheter positioned in the celiac trunk and superior mesenteric artery with an automated injector. Once the decision of applying empiric strategy to embolize the suspected bleeding vessel was made by the surgeons, the interventional radiologists would perform the procedure based on the following indications to prevent hepatic infarction and secure safety: embolization was expected to be technically feasible, and hepatic blood flow by an alternative route was confirmed. Embolization was performed with super-selective catheterization. Coils were applied to obtain complete occlusion, with both distal and proximal to the suspected bleeding site included.

Two different strategies, empiric and conservative, were applied in the interventional treatment of post PD hemorrhage for these 21 patients. An empiric group was defined as the cases underwent embolization despite negative angiographic outcomes, while a conservative group was defined as those who received medical therapy after the angiography. Clinical data, including age, sex, pathological diagnosis, lab test results, clinical presentation and onset time of bleeding after surgery, technical and clinical outcome were obtained from the medical records. For this study follow up was completed once the patient died or at least 1 month after the discharge.

\section{Results}

21 patients with negative angiographic results were enrolled in our study. There were 14 males and 7 females. The patients' ages ranged from 47 to 77 years (median age 60 years). Pathological examination of surgical specimens revealed pancreatic adenocarcinoma in 9 cases, cholangiocarcinoma in 6 , duodenal adenocarcinoma in 3 , ampullary adenocarcinoma in 1 , pancreatic mucinous carcinoma in 1 , duodenal neuroendocrine carcinoma in 1. 
All patients in our series presented with delayed $\mathrm{PPH}$, defined as hemorrhage occurring more than 24 hours postoperatively. Duration from the PD to the onset of hemorrhage ranged from 4 to 50 days, with a median of 17 days. Their clinical characteristics were summarized in Table 1. 
Table 1

Profiles of 21 cases underwent angiography for PPH with negative results

\begin{tabular}{|c|c|c|c|c|}
\hline Case & $\begin{array}{l}\text { Time until } \\
\text { bleeding (d) }\end{array}$ & Location & $\begin{array}{l}\text { Embolization } \\
\text { site }\end{array}$ & Clinical outcome \\
\hline \multicolumn{5}{|c|}{ Empiric group (12 cases) } \\
\hline 1 & 15 & extraluminal & $\mathrm{HA}$ & Died of bleeding 1 day later \\
\hline 2 & 14 & extraluminal & $\mathrm{HA}$ & \\
\hline 3 & 14 & extraluminal & $\mathrm{HA}$ & \\
\hline 4 & 17 & extraluminal & $\mathrm{HA}$ & \\
\hline 5 & 7 & extraluminal & $\mathrm{HA}$ & \\
\hline 6 & 15 & intraluminal & $\mathrm{HA}$ & Relap for re-bleeding18 days later \\
\hline 7 & 13 & extraluminal & $\mathrm{HA}$ & \\
\hline 8 & 14 & intraluminal & $H A+L G A$ & \\
\hline 9 & 22 & intraluminal & $\mathrm{HA}$ & \\
\hline 10 & 12 & extraluminal & $\mathrm{HA}$ & Relap for re-bleeding 4 days later \\
\hline 11 & 26 & intraluminal & $\mathrm{HA}$ & \\
\hline 12 & 21 & intraluminal & LGA & Died of re-bleeding 9 days later \\
\hline \multicolumn{5}{|c|}{ Conservative group (9 cases) } \\
\hline 13 & 24 & intraluminal & & \\
\hline 14 & 23 & intraluminal & & Relap for re-bleeding 6 days later \\
\hline 15 & 35 & extraluminal & & \\
\hline 16 & 50 & intraluminal & & \\
\hline 17 & 20 & extraluminal & & $\begin{array}{l}\text { Re-angiography and embolized HA } 10 \\
\text { ds later }\end{array}$ \\
\hline 18 & 4 & intraluminal & & \\
\hline 19 & 12 & intraluminal & & \\
\hline 20 & 32 & intraluminal & & $\begin{array}{l}\text { Re-lap for re-bleeding 7days later, but } \\
\text { died }\end{array}$ \\
\hline 21 & 45 & intraluminal & & \\
\hline
\end{tabular}

In the empiric group, embolization was performed at the hepatic artery in 11 cases and at the left gastric artery (LGA) in 1. Two patients died during the follow up. One died of hemorrhage recurrence despite 
embolization of the hepatic artery the same day after the interventional procedure. The other had bleeding recurrence 7 days after the LGA embolization and received endoscopic hemostasis, but this case still died of hemorrhage 2 more days later. Two patients required laparotomy and recovered in this group. One underwent abscess debridement for hemorrhage relapse 18 days after the embolization. The other patient underwent exploratory laparotomy and bleeding from the gastroepiploic artery was found and ligated 4 days after the embolization. No evidence of liver disfunction or other severe complication caused by embolization in this group. Recurrence rate was $33.3 \%(4 / 12)$ in this group and mortality rate was $16.7 \%(2 / 12)$.

In the conservative group, one patient required re-angiography with bleeding from the hepatic artery revealed 10 days after the first angiography. Hepatic artery embolization was performed and pancreaticojejunostomy fistula was confirmed and repaired during the subsequent relaparotomy. Another two patients required relaparotomy for hemorrhage recurrence 2 and 6 days respectively. Bleeding from the hepatic artery (Fig. 1-3) and the gastroduodenal artery (GDA) stump was revealed and ligated respectively in the two cases. No patient died of hemorrhage in this group during the follow up. Recurrence rate was 33.3\% (3/9) in this group and all these 3 cases required relaparotomy as definite treatment.

\section{Discussion}

Post PD hemorrhage is a life-threatening post PD complication that requires prompt and appropriate treatment. It was reported to be one of the most common causes of death within 90 days of PD (15). The development of pancreatic fistula and related abdominal abscess formation are the main cause of erosion of major vessels and thus of delayed bleeding episodes $(16,17)$.

Negative angiography that provide no evidence of arterial extravasation or pseudoaneurysm poses a dilemma situation since it does not exclude the potential threat of subsequent massive bleeding (17). The false-negative angiographies could be due to the intermittent character of bleeding episodes, spastic contraction of blood and/or obstruction of vessels or blood clots $(9,10,18)$. Therefore, empiric embolization of suspected vessels based on high clinical suspicion were supported (13). Similar recurrence rate $(33.3 \%)$ in both groups was demonstrated in our study.

Conservative medical therapy carries a great risk of massive hemorrhage that even had no chance for repeated interventional procedure, while radical embolization may result in liver necrosis and intrahepatic abscess as well as to the necrosis of the extrahepatic bile duct or bilioenteric anastomosis (17). In the case of negative angiography, Bhavraj S. Khalsa et al performed either empiric embolization of the suspected site of bleeding, or vascular access was maintained in the common femoral artery during the following 24 hours in case of rebleed (12). Yekebas EF et al also chose to maintain vascular access for 24 hours when angiography failed to localize the bleeding site, and patients underwent re-angiography in case of recurrent bleeding within this period. The empiric embolization was successful in 4 among 5 patients with negative angiographic visualization based on the surgeons' suspicion according to their 
study (13). However, 24 hours was obviously not long enough for hemorrhage recurrence observation in our study, since the recurrence time was all over 24 hours in the 5 cases with bleeding relapse (Table 1).

Determining empiric embolization is complex, and there are currently no acceptable criteria. General factors to consider when assessing suitability for embolization include hemorrhage severity, rebleeding risk, and whether the hepatic blood flow by an alternative route exist. The clinical decision of embolization was up to the hepatobiliary surgeon when negative result of angiography in our center, while the interventional radiologists determined the feasibility of the embolization. Hopefully, some early postoperative CT findings might play a critical role in predicting late post PD hemorrhage as that had been suggested by Han GJ et al (19).

Our data suggest that empiric embolization despite negative angiography result carries no obvious decreased recurrence rate, but its impact on survival warrant further study in larger samples. The two deaths in the empiric group might be partly explained by the high risk in emergency embolization due to hemodynamic coagulopathy when the patients had already experienced significant blood loss. The surgeons' bias might exist when they decide empiric strategy for severe hemorrhage.

The recurrence rate in both groups in this series reminded us that embolization is still a temporizing measure in the presence of sepsis and pancreatic leak. Continued vascular damage can lead to recurrence of bleeding from the same site or other sites $(5,9)$. Surgery is the only option to control bleeding and manage the primary causes simultaneously (18). However, scheduled operation can be safe with reliable embolization performed. Empiric embolization seemed to bridge some patients for subsequent relaparotomy, with patients' condition stable to endure the operation in this study.

The presented study has several limitations. Firstly, the sample size was small, without enough sample size for contrast study. Secondly, the retrospective nature of the study. Thirdly, subjective bias must exist since clinical decision was mainly made by the surgeons, based on their surgical experience and clinical suspicion. And obviously empiric embolization is preferred for patients with higher bleeding recurrence risk according to the surgeons' judgement. Further cohort contrast study with larger sample size is warranted. Finally, CT findings of the negative cases were not included in this study.

\section{Conclusions}

In conclusion, prompt decision making is required when negative result demonstrated during the angiography for post PD hemorrhage, and the surgeons' judgement is mandatory. This single center study indicated that both empiric and conservative treatment may be effective as indicated when negative angiography presented. Great caution is required following the interventional procedure, because recurrence rate after both treatment methods is significant despite negative angiography.

\section{Abbreviations}

PD: pancreaticoduodenectomy; LGA: left gastric artery; GDA: gastroduodenal artery 


\section{Declarations}

\section{Ethics approval and consent to participate}

The need for written ethics approval for retrospective study was waived in our Beijing Chaoyang Hospital. The statement from the ethics committee for waiving approval of this retrospective study can be available for review by the request.

\section{Consent for publication}

Written informed consent was obtained from the participants for publication of this article and any accompanying images. A copy of the written consent is available for review by the Editor of this article.

\section{Availability of data and materials}

Not applicable.

\section{Competing interests}

The authors declare that they have no competing interests.

\section{Funding}

This retrospective study did not receive any specific grant from funding agencies in the public, commercial, or not-for-profit sectors.

\section{Authors' contributions}

Q H, K G, and J-F W have all made substantial contributions to conception, acquisition of data, analysis and interpretation of data. All of them have been involved in drafting the manuscript and revising for the content. All authors read and approved the final manuscript and take public responsibility for appropriate portions of the content and agreed to be accountable for all aspects of work.

\section{Acknowledgements}

The participation of Bao-Jie Wei, Chuan-Guo Zhou, and Hui Li, for completion of the clinical work is greatly appreciated.

\section{References}

1. Asari S, Matsumoto I, Toyama H, et al. Recommendation of treatment strategy for postpancreatectomy hemorrhage: Lessons from a single-center experience in 35 patients. Pancreatology. 2016; 16(3): 454-63.

2. Gao F, Li J, Quan S, et al. Risk Factors and Treatment for Hemorrhage after Pancreaticoduodenectomy: A Case Series of 423 Patients. Biomed Res Int. 2016; 2016: 2815693. 
3. Welsch T, Eisele H, Zschäbitz S, et al. Critical appraisal of the international study group of pancreatic surgery (ISGPS) consensus definition of postoperative hemorrhage after pancreatoduodenectomy. Langenbecks Arch Surg. 2011; 396(6): 783-91.

4. Muglia R, Lanza E, Poretti D, et al. Emergency endovascular treatments for delayed hemorrhage after pancreaticobiliary surgery: indications, outcomes, and follow-up of a retrospective cohort. Abdom Radiol (NY). 2020 Mar 14. doi: 10.1007/s00261-020-02480-z.

5. Puppala S, Patel J, McPherson S, et al. Hemorrhagic complications after Whipple surgery: imaging and radiologic intervention. AJR Am J Roentgenol. 2011; 196(1): 192-7.

6. Wolk S, Grützmann R, Rahbari NN, et al. Management of clinically relevant postpancreatectomy hemorrhage (PPH) over two decades - A comparative study of 1450 consecutive patients undergoing pancreatic resection. Pancreatology. 2017; 17(6): 943-950.

7. Zhou TY, Sun JH, Zhang YL, et al. Post-pancreaticoduodenectomy hemorrhage: DSA diagnosis and endovascular treatment. Oncotarget. 2017; 8(43): 73684-73692.

8. Stampfl U, Hackert T, Sommer CM, et al. Superselective embolization for the management of postpancreatectomy hemorrhage: a single-center experience in 25 patients. J Vasc Interv Radiol. 2012; 23(4): 504-10.

9. Roulin D, Cerantola Y, Demartines N, et al. Systematic review of delayed postoperative hemorrhage after pancreatic resection. J Gastrointest Surg. 2011; 15(6): 1055-62.

10. Liu C, Qiu YH, Luo XJ, et al. Treatment of massive pancreaticojejunal anastomotic hemorrhage after pancreatoduodenectomy. World J Gastroenterol. 2009; 15(13): 1625-9.

11. Izumo W, Higuchi R, Yazawa T, et al. Evaluation of preoperative risk factors for postpancreatectomy hemorrhage. Langenbecks Arch Surg. 2019; 404(8): 967-974.

12. Khalsa BS, Imagawa DK, Chen JI, et al. Evolution in the treatment of delayed postpancreatectomy hemorrhage. Pancreas. 2015; 44(6): 953-8.

13. Yekebas EF, Wolfram L, Cataldegirmen G, et al. Postpancreatectomy hemorrhage: diagnosis and treatment: an analysis in 1669 consecutive pancreatic resections. Ann Surg. 2007; 246(2): 269-80.

14. Wente MN, Veit JA, Bassi C, et al. Postpancreatectomy hemorrhage (PPH): an International Study Group of Pancreatic Surgery (ISGPS) definition. Surgery. 2007; 142(1): 20-5.

15. Narayanan S, Martin AN, Turrentine FE, et al. Mortality after pancreaticoduodenectomy: assessing early and late causes of patient death. J Surg Res. 2018; 231: 304-308.

16. Uggeri F, Nespoli L, Sandini M, et al. Analysis of risk factors for hemorrhage and related outcome after pancreatoduodenectomy in an intermediate-volume center. Updates Surg. 2019; 71(4): 659-667.

17. Lee JH, Hwang DW, Lee SY, et al. Clinical features and management of pseudoaneurysmal bleeding after pancreatoduodenectomy. Am Surg. 2012; 78(3): 309-17.

18. Feng F, Cao X, Liu X, et al. Two forms of one complication: Late erosive and nonerosive postpancreatectomy hemorrhage following laparoscopic pancreaticoduodenectomy. Medicine (Baltimore). 2019; 98(30): e16394. 
19. Han GJ, Kim S, Lee NK, et al. Prediction of late postoperative hemorrhage after Whipple procedure using computed tomography performed during early postoperative period. Korean J Radiol. 2018; 19(2): 284-291.

\section{Figures}

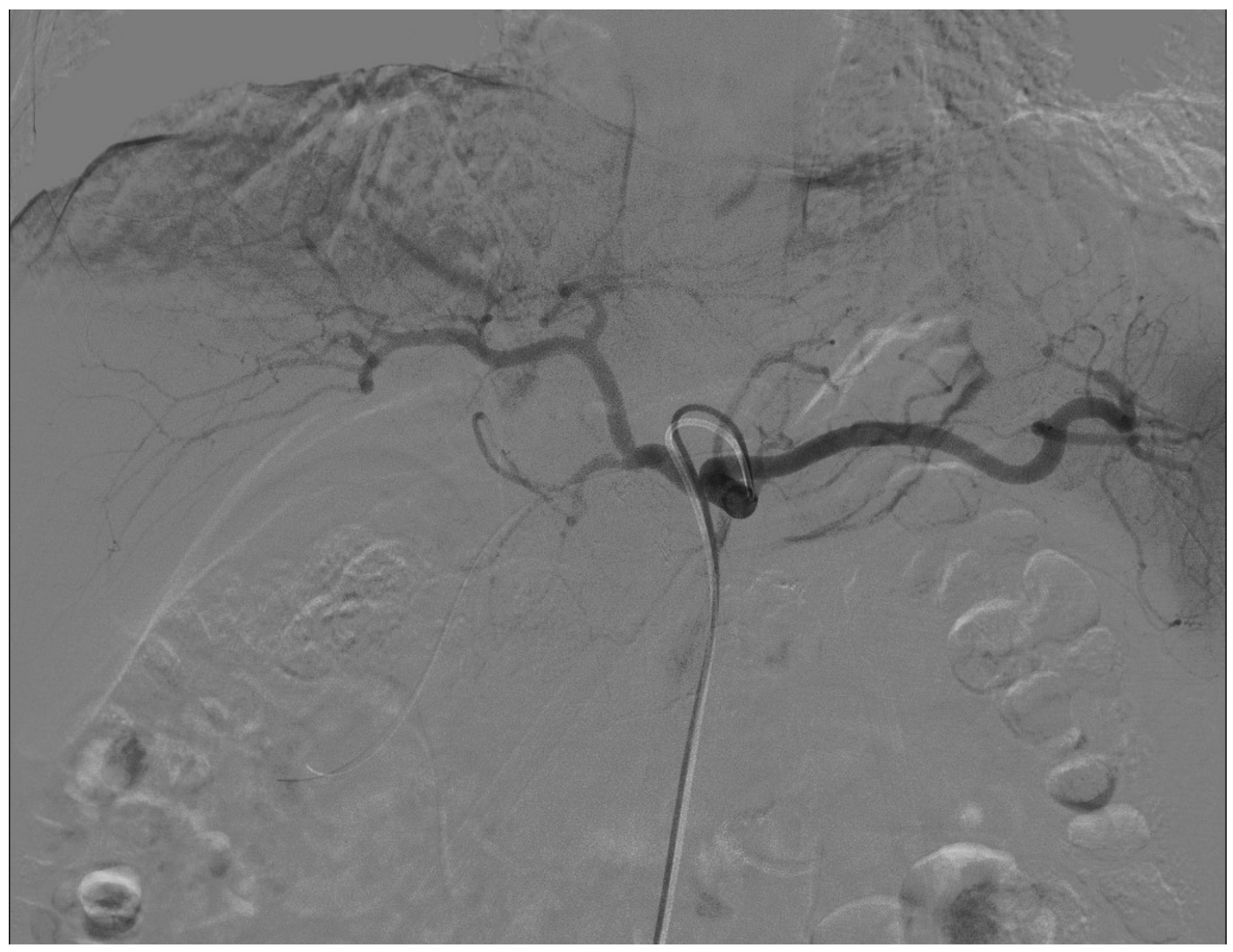

\section{Figure 1}

A 47-year-old man had angiography 20 days after PD because of the hemorrhage into the drainage catheter. There was no obvious sign of bleeding in the first angiography and conservative treatment was applied since the patient was hemodynamically stable. 


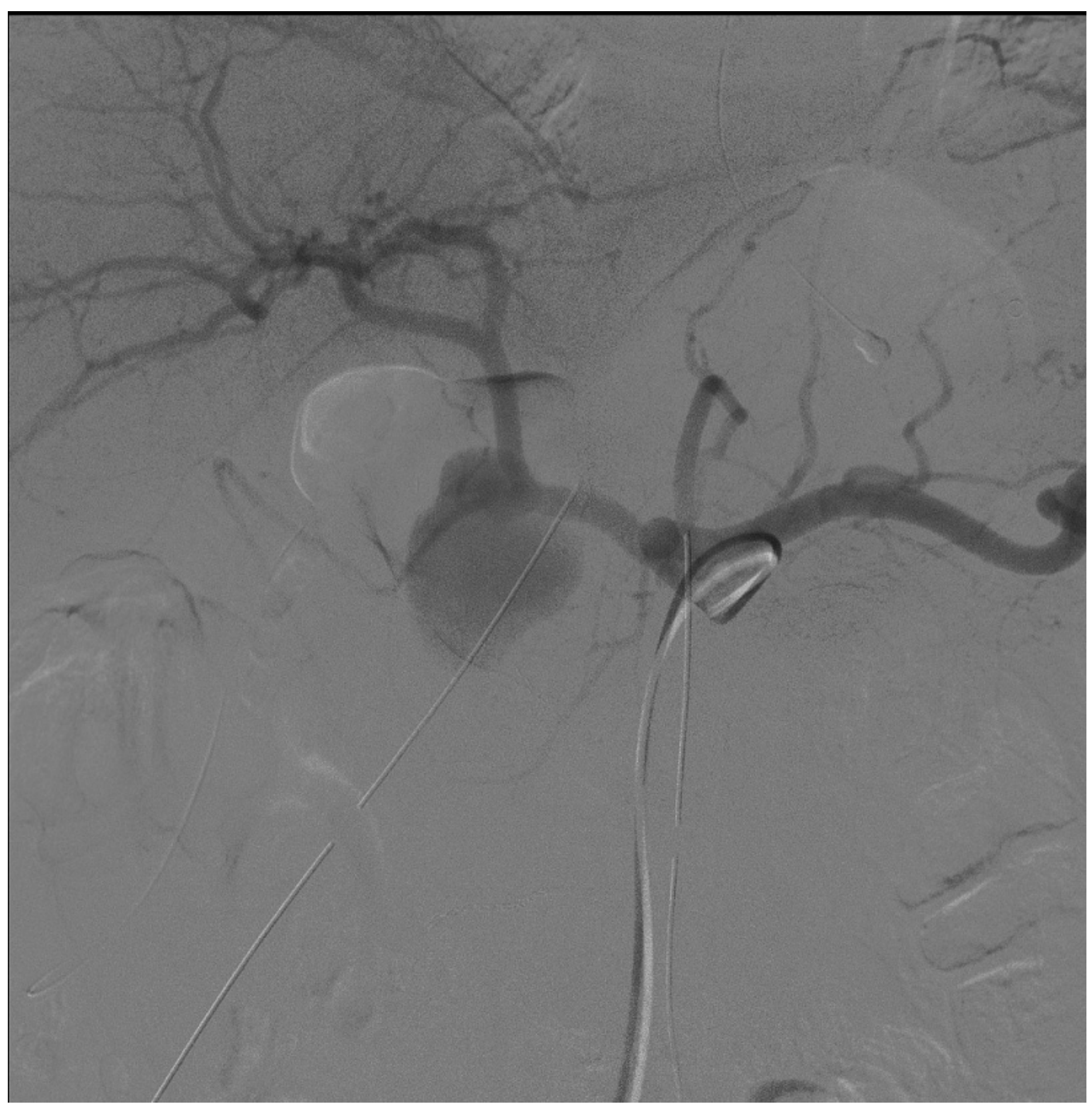

\section{Figure 2}

The same patient required emergency repeated angiography because of the recurrence of hemorrhage 10 days after the first angiography. The pseudoaneurysm of the hepatic artery was confirmed. 


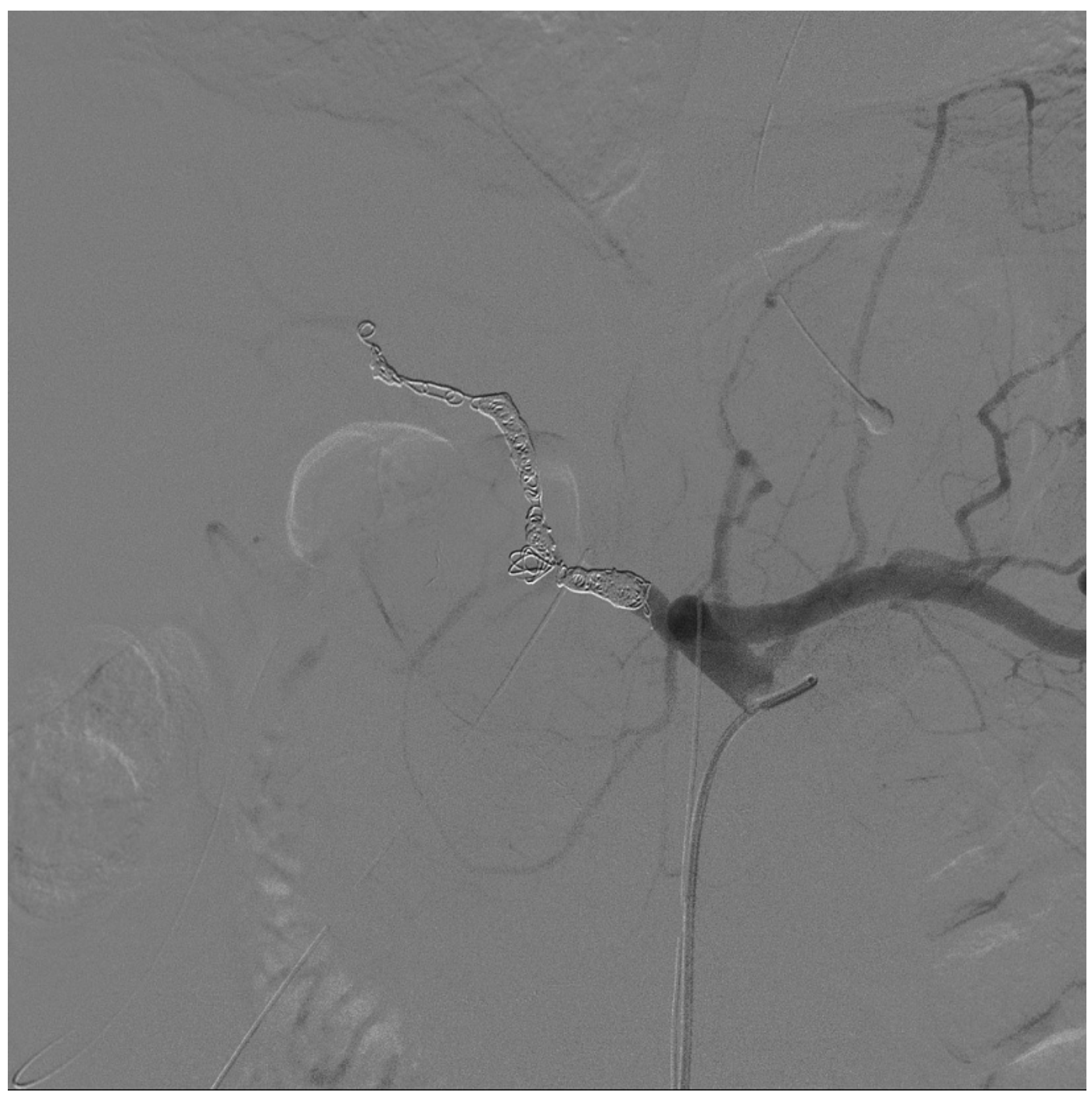

\section{Figure 3}

Embolization was performed successfully to achieve hemostasis. The anastomotic dehiscence was confirmed and sutured during the relaparotomy 2 days after the embolization. 